\title{
Statistical Analysis for the Q-factor of twin $\mathrm{kHz}$ QPOs
}

\author{
J. Wang ${ }^{1, \star}$, H. K. Chang ${ }^{1}$, C. M. Zhang ${ }^{2}$, D. H. Wang ${ }^{3,2}$ and L. Chen ${ }^{3}$ \\ 1 Institute of Astronomy, National Tsing Hua University, Hsinchu, 30013, Taiwan \\ 2 National Astronomical Observatories, Chinese Academy of Sciences, Beijing 100012, P. R. China \\ 3 Astronomy Department, Beijing Normal University, Beijing, 100875, China
}

Received XXXX, accepted XXXX

Published online XXXX

Key words accretion: accretion disks-stars: neutron-binaries: close-X-rays: stars-pulsar.

Using the recently published data of twin kHz quasi-period oscillations (QPOs) in neutron star low-mass X-ray binaries (LMXBs), we study the different profiles between bright $\mathrm{Z}$ sources and less luminous Atoll sources in a statistical way. We find the quality factors of upper kHz QPOs show a narrow distribution both for Z sources and Atoll sources, which concentrate at 7.98 and 9.75 respectively, the quality factors of lower $\mathrm{kHz}$ QPOs show a narrow distribution for $\mathrm{Z}$ sources and a broader distribution for Atoll sources, which concentrate at 5.25 and 86.22 respectively. In order to investigate the relation between the quality factor and the peak frequency of $\mathrm{kHz}$ QPOs, we fit the data with power-law, linear and exponential functions, respectively. There is an obvious trend that the quality factors increase with the peak frequencies both for upper and lower QPOs. The implications of our results are discussed.

Copyright line will be provided by the publisher

\section{Introduction}

A number of neutron stars in low mass X-ray binaries show the kilohertz quasi-periodic oscillations in their X-ray spectra (van der Klis 2000, 2006). These frequencies, in the range of $200 \sim 1300 \mathrm{~Hz}$, are as the same order as the dynamical timescale of the innermost region of the accretion flow around the stellar mass compact objects (van der Klis 2006, 2008). Owing to the expected links with the orbital motion, most work about $\mathrm{kHz}$ QPOs focus on the explanation for the nature of these signals (e.g. Miller, Lamb \& Psaltis 1998; Stella \& Vietri 1998, 1999; Kluzniak \& Abramowicz 2001; Abramowicz et al. 2003; Zhang 2004). The kHz QPOs often arise as simultaneous twin peaks (upper $\nu_{2}$ and lower $\nu_{1}$ frequency) with frequencies changing over time. These frequencies behave in a rather regular way and follow the tight correlations between their frequencies and other observed characteristic frequencies (see, e.g. Psaltis et al. 1998, 1999a; Psaltis, Belloni \& van der Klis 1999b; Stella, Vietri \& Morsink 1999; Belloni, Psaltis \& van der Klis 2002; Titarchuk \& Wood 2002; Mendez \& van der Klis 1999, 2000; Méndez et al. 2001; Yu, van der Klis, Jonker 2001; Yu, van der Klis 2002). Moreover, the correlation between the upper frequency and the lower frequency across different sources can be roughly fitted by a powerlaw function (see e.g. Psaltis et al. 1998, 1999a; Zhang et al. 2006), and also by a linear model (see Belloni, Méndez \& Homan 2005, 2007).

The kHz QPOs in LMXBs are narrow features (peaks) in their power density spectra (PDS), whose profiles can be described by the Lorentzian function $P_{\nu} \propto A_{0} w /[(\nu-$

^ Corresponding author: joanwangj@126.com $\left.\left.\nu_{0}\right)^{2}+(w / 2)^{2}\right]\left(\nu_{0}\right.$ is the peak frequency, $w$ is the full width at half-maximum (FWHM), and $A_{0}$ is the amplitude of the signal). The ratio of peak frequency to FWHM is the quality factor,

$$
Q \equiv \frac{\nu_{0}}{w}
$$

Therefore, the $\mathrm{kHz}$ QPOs in neutron star LMXBs can be characterized by three characteristic quantities, i.e. centroid frequency (i.e., peak frequency $\left.\nu_{0}\right)$, quality factor $\left(\mathrm{Q} \equiv \nu_{0} /\right.$ FWHM) and fractional root-mean-squared (rms). The quality factor characterizes the coherence of the signal, while the rms represents a measure of the signal strength and it is proportional to the square root of the peak power contribution to the PDS. Each $\mathrm{kHz}$ QPO corresponds to its quality factor (lower $Q_{1}$ and upper $Q_{2}$ quality factor) and centroid frequency (upper $\nu_{2}$ and lower $\nu_{1}$ frequency).

In the past several years, the large Rossi X-ray Timing Explorer (RXTE) archive makes it possible to study this quantity systematically in several sources. Using the data from RXTE, Barret et al. (2005a) studied the source $4 \mathrm{U} 1608-52$ and revealed a positive correlation between the lower frequency QPOs and its quality factors, up to a maximum of about $Q \sim 200$. Motivated by this idea, Barret, Olive \& Miller (2005b, 2006) studied the QPO properties and the dependency of its quality factor on the peak frequency in source $4 \mathrm{U}$ 1636-536. It shows that the quality factors for lower and upper kHz QPOs of 4U 1636-536 follow different tracks in a $Q$ vs. $\nu$ plot, i.e. the quality factors of lower $\mathrm{kHz}$ increase with its peak frequency up to $850 \mathrm{~Hz}(\mathrm{Q} \sim 200)$ and drop precipitously to the highest detectable frequency $\sim 920 \mathrm{~Hz}(\mathrm{Q} \sim 50)$, while the quality factors of upper $\mathrm{kHz}$ QPOs increase steadily all the way 
to the highest detectable frequency. Moreover, the quality factors of lower QPOs is higher than those of upper ones (Barret, Olive \& Miller 2005b,c; 2006). The rough similarity was also extended to sources 4U 1735-44 and 4U 172834 (Barret, Olive \& Miller 2006; Boutelier, Barret \& Miller 2009; Méndez 2006; Török 2009).

In this paper, we analyze the distributions of the quality factors for upper and lower $\mathrm{kHz}$ QPOs in ten sources — five Atoll sources (namely 4U 1608-52, 4U 1636-53, 4U 1728-34, 4U 1820-30 and 4U 1735-44) and five Z sources (namely Sco X-1, Cyg X-2, GX 17+2, GX 5-1 and GX $340+0)$. In order to investigate the relation between the quality factors and its centroid frequencies, we fit the data with the three functions, i.e. linear relation $(Q=a+b \nu / 1000)$, power-law relation $\left(Q=a(\nu / 1000)^{b}\right)$ and exponential relation $(Q=a \times \exp (b \nu / 1000))$, according to $Q-\nu$ tracks. We benefit from the existing studies and use the published data from the collection by Méndez (2006, and reference therein). In section 2, we analyze the data for these sources and execute fitting for the different source categories. Conclusions and discussions are contained in section 3.

\section{Statistical Analysis for the Coherence of kHz QPOs}

In this part, we investigate the data statistically and choose linear $(Q=a+b \nu / 1000)$, power-law $\left(Q=a(\nu / 1000)^{b}\right)$ and exponential relation $(Q=a \times \exp (b \nu / 1000))$ for fitting, where $a$ and $b$ are the undefined parameters. Firstly, we put the sources together and fit them with above three relations, then we divide them into Atoll and $\mathrm{Z}$ sources and execute the same fitting.

\subsection{Statistically Analysis for the Data}

Firstly, we study the distributions of upper and lower quality factors for $\mathrm{Z}$ and Atoll sources. Fig. 1 presents the distributions of upper quality factors $\left(Q_{2}\right)$ for $\mathrm{Z}$ and Atoll sources. It is found that most $Q_{2}$ of these two classes locate in the similar region (i.e. $Q_{2}=2$ - 18) as a whole. For the exception, a few $Q_{2}$ of Atoll sources present relatively larger values (up to 40, see Fig. 1 for detail). Most $Q_{2}$ for $\mathrm{Z}$ sources gather in a range from 2 to 10 which is larger than those of Atoll sources. For a quantitative knowledge, we calculate the mean value of $Q_{2}$ for $\mathrm{Z}$ and Atoll sources, i.e. $<$ $\left.Q_{2 \text { Atoll }}\right\rangle=9.75$ and $\left\langle Q_{2 Z}\right\rangle=7.98$. The mean value of $Q_{2}$ for $\mathrm{Z}$ sources is lower than that for Atoll sources.

In Fig. 2 we plot the distributions of lower quality factors $\left(Q_{1}\right)$ for $\mathrm{Z}$ and Atoll sources, where we notice very big difference in the range of $Q_{1}$ between these two sources. The $Q_{1}$ for $\mathrm{Z}$ sources are small $\left(Q_{1 Z} \leq 14\right)$, and those for Atoll sources are very large (up to $Q_{1}=200$ ). The $Q_{1}$ ranges from 2 to 14 for $Z$ sources and from 2 to 200 for Atoll sources (see Fig. 2 for detail). Most data of $Q_{1}$ for $\mathrm{Z}$ sources are distributed from 6 to 10, and that of Atoll sources gather in the region of $Q_{1} \sim 60-100$. Some $Q_{1}$ for Atoll sources

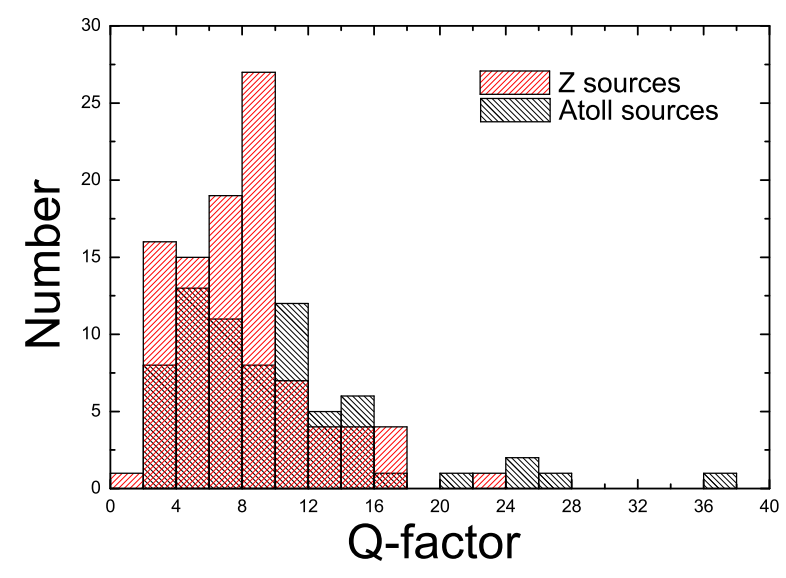

Fig. 1 The distributions of $Q_{2}$ for $\mathrm{Z}$ and Atoll sources. The red shadow represents the $Q_{2}$ distribution for $\mathrm{Z}$ sources, while the black shadow show that for Atoll sources. The mean value of $Q_{2}$ is 7.98 for Atoll sources and 9.75 for $\mathrm{Z}$ sources. For the plot, we take the width of bin as 2 .

are as high as 200. We also calculate the mean values of $Q_{1}$ both for $\mathrm{Z}$ and Atoll sources, i.e. $\left\langle Q_{1 \text { Atoll }}>=86.22\right.$, $<Q_{1 Z}>=5.25$.

On the whole, the quality factors for $\mathrm{Z}$ sources are smaller than those for Atoll sources. In addition, $Q_{2}$ for $\mathrm{Z}$ sources cover a wider range than $Q_{1}$, while $Q_{2}$ cover the narrower range than $Q_{1}$ for Atoll sources. The mean values of $Q_{1}$ and $Q_{2}$ are $<Q_{1}>=49.20$ and $<Q_{2}>=8.70$, respectively.

\subsection{Fitting for the $Q_{2}-\nu_{2}$ Relations}

In order to study the $Q_{2}-\nu_{2}$ relation, we use linear $(Q=$ $a+b \nu / 1000)$, power-law $\left(Q=a(\nu / 1000)^{b}\right)$ and exponential relations $(Q=a \times \exp (b \nu / 1000))$ to fit $Q_{2}-\nu_{2}$ trajectories for ten sources. It is noticed that the $Q_{2}$ values are similar for $\mathrm{Z}$ and Atoll sources, which increase with $\nu_{2}$, so we fit the three relations to total ten sources, $Z$ sources and Atoll sources, respectively (see Fig. 3). The values of fitting parameters ( $a$ and $b$ ) and the fitting results are listed in table 1 .

From Fig. 3 it is seen that the data points are dispersive in the left and right panels, while the result in the middle panel is better, especially for power-law and exponential relations. From table 1 we notice that the fitting results are not good for these large $\chi^{2} / d$.o.f.. If we just forecast a roughly trend based on these results, it can be seen that the powerlaw and exponential relations fit mildly better than that of linear relation as a whole. Exponential relation fits better than power-law and linear relations both for $\mathrm{Z}$ sources and for Atoll sources. The fittings for $Q_{2}-\nu_{2}$ of $\mathrm{Z}$ sources are better than that for Atoll sources, with relatively small reduced $\chi^{2}\left(\chi^{2} /\right.$ d.o.f. $=4.73,5.01$ and 5.76 for exponential, power-law and linear relations, respectively, see table 1 for 

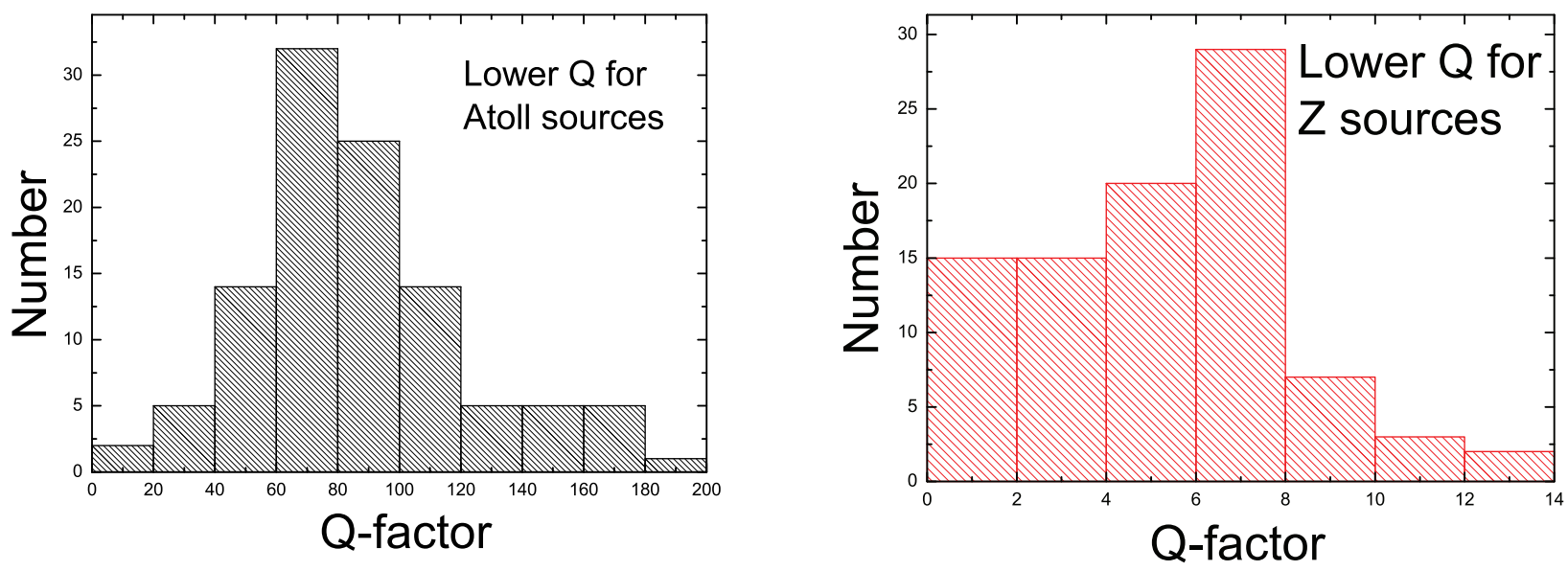

Fig. 2 The distributions of $Q_{1}$ for $\mathrm{Z}$ and Atoll sources. The left panel is for Atoll sources, and the right one is for $\mathrm{Z}$ sources. The mean value of $Q_{1}$ is 86.22 for Atoll sources and 5.25 for $\mathrm{Z}$ sources. For the plot, the width of bins are set to 20 and 2 for Atoll and $\mathrm{Z}$ sources, respectively.

detail). But for five Atoll sources, the reduced $\chi^{2}$ are relatively large $\left(\chi^{2} /\right.$ d.o.f. $=24.63,26.10$ and 29.33 for exponential, power-law and linear relation, respectively), and the exponential fitting is also better than the other two relations. If we put all the sources together and fit three relations to them, we find that the power-law relation with a small $\chi^{2} /$ d.o.f. (11.75) is better than the other two.

\subsection{Fitting for the $Q_{1}-\nu_{1}$ Relations}

Following the same techniques, we investigate the $Q_{1}-\nu_{1}$ relations for $\mathrm{Z}$ and Atoll sources (see Fig. 4).

The left panel of Fig. 4 show the results for $\mathrm{Z}$ sources, in which it can be seen that almost all points form the rising trend, and we fit power-law, linear and exponential relations to all the data points, respectively. The parameters and fitting results are listed in table 2. The reduced $\chi^{2}$ are 3.33, 3.30 and 3.62 for power-law, linear and exponential relation, respectively. It seems that the linear relation fits better than the other two.

The right panel of Fig. 4 4 show the results for Atoll sources, in which it can be seen that the $Q_{1}-\nu_{1}$ distribution of five Atoll sources display two rising parts and two dropping parts (see the right panel of Fig. 4 for detail). We take the sources 4U 1608-52 and 4U 1820-30, which have large $Q_{1}$ values, as a group. While the sources $4 \mathrm{U}$ 1636-53, $4 \mathrm{U}$ 1728-34 and $4 \mathrm{U}$ 1735-44, which have small $Q_{1}$ values, as the other group. Then we fit the data of the two groups respectively, the fitting results are in table 2 Compared with the fitting result of $Q_{2}-\nu_{2}$, the reduced $\chi^{2}$ is very large (see table 2 for detail), but also with large $R^{2}$. It is found that the linear relation fits better than the other two for the curve with relatively low $Q_{1}$ values (formed by $4 \mathrm{U} 1636$ 53, $4 \mathrm{U} 1728-34$ and $4 \mathrm{U} 1735-44$ ), and the power-law rela- tion fits better to the curve with high $Q_{1}$ values (formed by $4 \mathrm{U} 1608-52$ and $4 \mathrm{U} 1820-30$ ).

So far, there is no appropriate model which can explain the mechanism of lower $\mathrm{kHz}$ QPOs, as well as that of the lower quality factors. However, it is claimed that the lower $\mathrm{kHz}$ QPOs arise in the innermost region of accretion disk (van der Klis 2006). The quality factors relate to the frequency drift in the inner disk (Barret, Olive \& Miller 2006; Wang et al. 2011). So we claim that the diverge of the data points between different sources may result from different physical environment of inner disk in different sources. In addition, the occurrence of drops in $Q_{1}-\nu_{1}$ plots for Atoll sources may arise from the existence of the inner boundary of accretion disk (Barret, Olive \& Miller 2006). Due to the different mechanism of the drops of $Q_{1}-\nu_{1}$ tracks for Atoll sources, we just fit the rising branches of the $Q_{1}-\nu_{1}$ track and do not consider the dropping parts with the same techniques.

\section{Discussions and Summary}

In this work, we study the distribution of the quality factors and the relation between the quality factor and peak frequency of $\mathrm{kHz}$ QPOs . We notice that there are differences in the distribution of the quality factors between lower and upper $\mathrm{kHz} \mathrm{QPOs}$, as well as $\mathrm{Z}$ and Atoll sources. By fitting the power-law, linear and exponential relation to the $Q_{\nu}$ tracks, we investigate the $Q_{\nu}$ relations for upper and lower kHz QPOs.

(1). The $Q_{2}$ values are low $\left(Q_{2}<18\right.$ in general, with a few exceptions) for both $\mathrm{Z}$ and Atoll sources. The $Q_{1}$ values for five $\mathrm{Z}$ sources are very low, $Q_{1 z}=2-14$. However, the $Q_{1}$ values for five Atoll sources are very high, and the maximum $Q_{1 \mathrm{Atoll}}$ is up to 200 (4U 1608-52). 

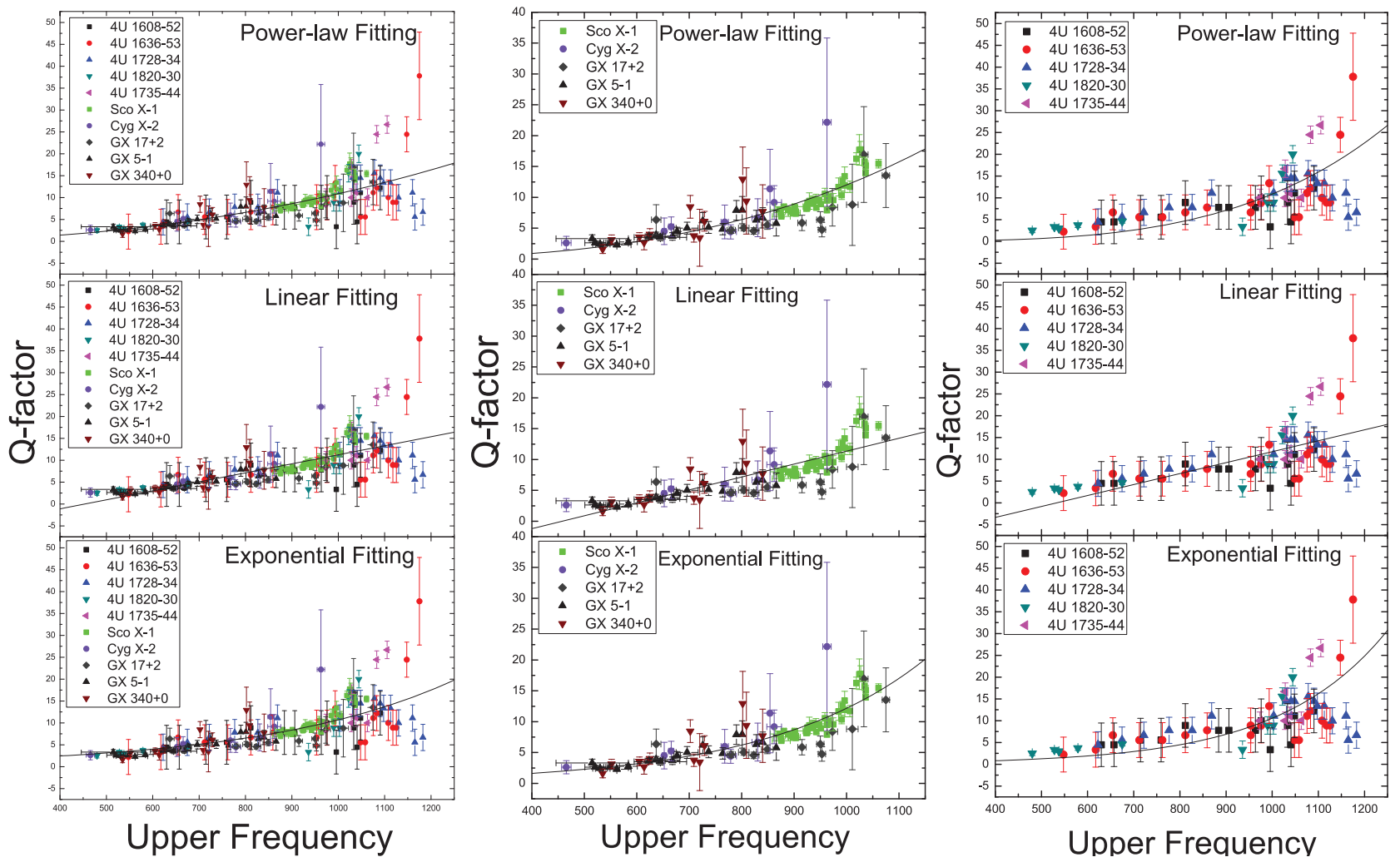

Fig. 3 The fitting plots of Q vs. $\nu$ for upper kHz QPOs. The left panel is for all ten sources. The middle (right) panel is for five $\mathrm{Z}$ (Atoll) sources. The three parts from top to bottom of each panel present the power, linear and exponential fitting, respectively. The point with different shapes and colors in the plots represent the different sources as shown in the legends. The fitting functions and results are shown in table 1

Table 1 The fitting results for quality factors of upper kHz QPOs correspond to Fig.3 .

\begin{tabular}{ccccc}
\hline Function & $\mathrm{a}$ & $\mathrm{b}$ & $\chi^{2} / D o F$ & $R^{2}$ \\
\hline Total fitting & & & & \\
$Q_{2}=a+b \nu_{2} / 1000$ & $-9 \pm 2$ & $21 \pm 2$ & 14.00 & 0.47 \\
$Q_{2}=a\left(\nu_{2} / 1000\right)^{b}$ & $10.9 \pm 0.3$ & $2.2 \pm 0.2$ & 11.75 & 0.49 \\
$Q_{2}=a \times \exp \left(b \nu_{2} / 1000\right)$ & $0.9 \pm 0.2$ & $2.5 \pm 0.2$ & 11.80 & 0.48 \\
\hline Z source & & & & \\
$Q_{2}=a+b \nu_{2} / 1000$ & $-10 \pm 1$ & $21 \pm 2$ & 5.76 & 0.64 \\
$Q_{2}=a\left(\nu_{2} / 1000\right)^{b}$ & $12 \pm 0.4$ & $2.8 \pm 0.3$ & 5.01 & 0.69 \\
$Q_{2}=a \times \exp \left(b \nu_{2} / 1000\right)$ & $0.4 \pm 0.1$ & $3.4 \pm 0.3$ & 4.73 & 0.71 \\
\hline Atoll source & & & & \\
$Q_{2}=a+b \nu_{2} / 1000$ & $-13 \pm 5$ & $25 \pm 5$ & 29.33 & 0.36 \\
$Q_{2}=a\left(\nu_{2} / 1000\right)^{b}$ & $10.9 \pm 0.9$ & $4 \pm 0.8$ & 26.10 & 0.43 \\
$Q_{2}=a \times \exp \left(b \nu_{2} / 1000\right)$ & $0.1 \pm 0.1$ & $4.3 \pm 0.8$ & 24.63 & 0.46 \\
\hline
\end{tabular}

(2). On the whole, the ranges of $\mathrm{Q}$ for five Atoll sources are wider than that for five $\mathrm{Z}$ sources both for upper and lower Q. According to the idea that the twin $\mathrm{kHz}$ QPOs come from the inner region of accretion disk, the instabilities which can lead to the radial drift and frequency drift in this region for Atoll sources are stronger than that for $\mathrm{Z}$ sources (Wang et al. 2011).

(3). From Fig. 2 it seems that the range of $Q_{1}$ for Atoll sources is about 10 times wider than that for $\mathrm{Z}$ sources. In addition, the high luminosity $\mathrm{Z}$ sources exhibit luminosity close to critical Eddington luminosity $L_{E d d}$, and the range of their luminosity is $0.5-1 L_{E d d}$. But Atoll sources present low luminosity $0.001-0.2 L_{E d d}, 100$ times lower than that for Z sources (Hasinger and van der Klis 1989; Hasingger 1990, see van der Klis 2006 for a review). Therefore, we expect a relation of $Q_{1} \sim L^{-1 / 2}$ between the luminosity and quality factors for lower $\mathrm{kHz}$ QPOs to be investigated and proven in future.

(4). The fitting results of $Q_{2} v s . \nu_{2}$ can not confirm the actual relation between $Q_{2}$ and $\nu_{2}$ because of the large $\chi^{2} /$ d.o.f.. 

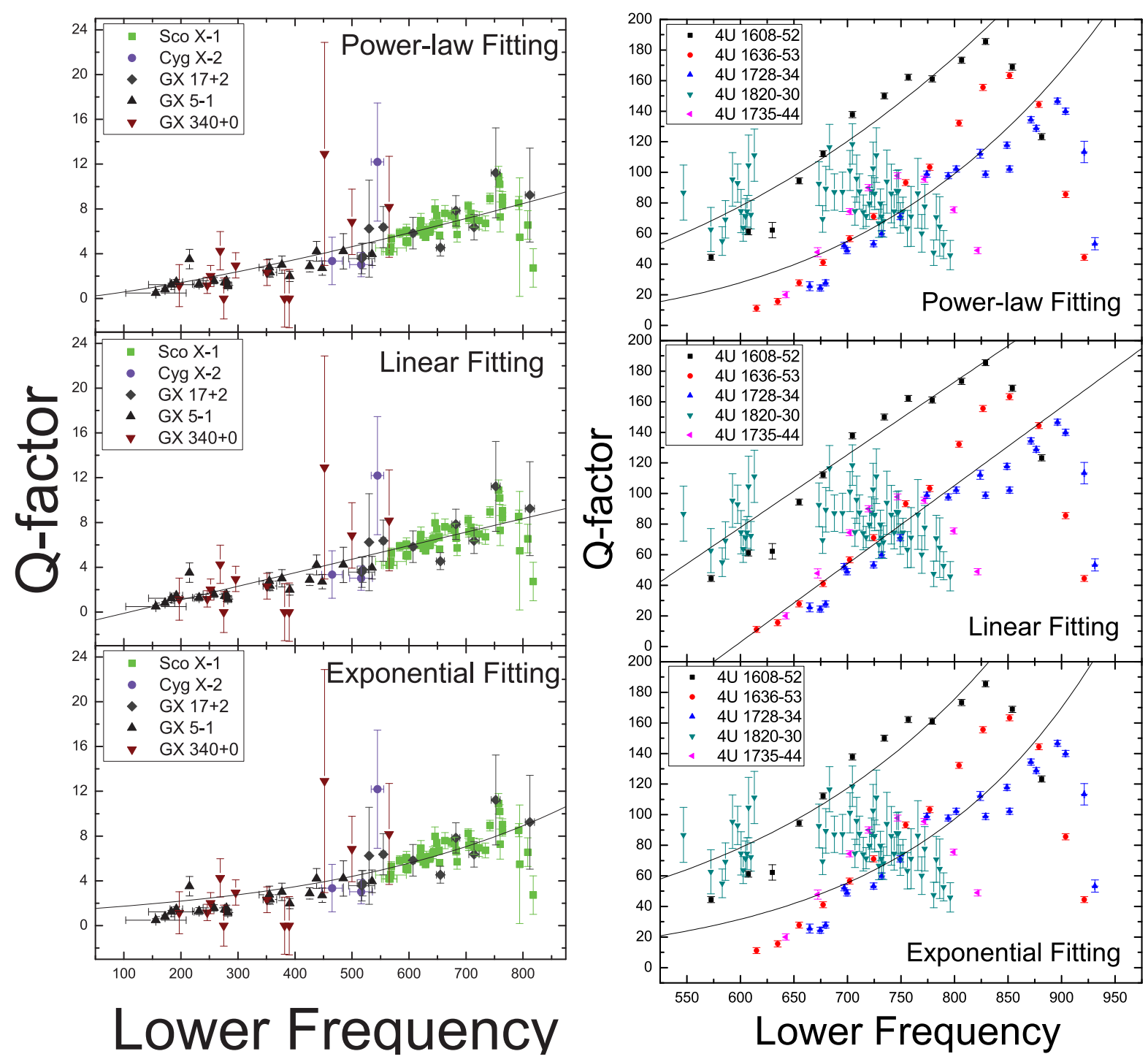

Fig. 4 The similar pattern as Fig. 3, but for lower kHz QPOs. The left panel is for five Z sources. The right panel is for five Atoll sources, in which we take the sources 4U 1608-52 and 4U 1820-30 (higher quality factors) as a group and the sources $4 \mathrm{U}$ 1636-53, 4U1728-34 and 4U 1735-44 (lower quality factors) as the other group, then we fit the data of two groups, respectively. The fitting functions and results are shown in Table.2

Table 2 The fitting results for quality factors of lower $\mathrm{kHz}$ QPOs correspond to Fig.4.

\begin{tabular}{ccccc}
\hline Function & $\mathrm{a}$ & $\mathrm{b}$ & $\chi^{2} / D o F$ & $R^{2}$ \\
\hline Z source & & & & \\
$Q_{1}=a+b \nu_{1} / 1000$ & $-1.3 \pm 0.6$ & $12 \pm 1$ & 3.30 & 0.59 \\
$Q_{1}=a\left(\nu_{1} / 1000\right)^{b}$ & $11.3 \pm 0.9$ & $1.3 \pm 0.2$ & 3.33 & 0.59 \\
$Q_{1}=a \times \exp \left(b \nu_{1} / 1000\right)$ & $1.4 \pm 0.3$ & $2.4 \pm 0.3$ & 3.62 & 0.56 \\
\hline Atoll source with lower $Q_{1}$ & & & & \\
$Q_{1}=a+b \nu_{1} / 1000$ & $-300 \pm 30$ & $500 \pm 30$ & 241.70 & 0.87 \\
$Q_{1}=a\left(\nu_{1} / 1000\right)^{b}$ & $260 \pm 30$ & $4.4 \pm 0.4$ & 343.75 & 0.82 \\
$Q_{1}=a \times \exp \left(b \nu_{1} / 1000\right)$ & $1.1 \pm 0.5$ & $5.6 \pm 0.6$ & 380.99 & 0.80 \\
\hline Atoll source with higher $Q_{1}$ & & & & \\
$Q_{1}=a+b \nu_{1} / 1000$ & $-200 \pm 30$ & $480 \pm 40$ & 270.52 & 0.85 \\
$Q_{1}=a\left(\nu_{1} / 1000\right)^{b}$ & $330 \pm 30$ & $2.8 \pm 0.2$ & 267.59 & 0.85 \\
$Q_{1}=a \times \exp \left(b \nu_{1} / 1000\right)$ & $7 \pm 2$ & $4 \pm 0.3$ & 279.81 & 0.84 \\
\hline
\end{tabular}


We consider that it may result from the small sample with the relative big errors. There is an obvious trend that $Q_{2}$ increases with $\nu_{2}$ for both $\mathrm{Z}$ and Atoll sources, and the powerlaw and exponential relations fit better than the linear relation.

(5). The fitting results of $Q_{1}$ vs. $\nu_{1}$ also cannot be considered as perfect. Then, an obvious trend is that the $Q_{1}$ increase with $\nu_{1}$ in the case of five $\mathrm{Z}$ sources, and the linear relation fits better than the other two.

(6). In the $Q_{1}-\nu_{1}$ diagrams for Atoll sources, all five sources present drops at a maximum $Q_{1}$. It is considered as the results of innermost boundary (ISCO) of accretion disk, firstly proposed by Barret and coauthors (see, Barret et al. $2005 b, 2005 c, 2006,2007)$. The special mechanism of the drops are expected to be investigated further. Here, we just fit the three relations to the rising parts.)

(7). As for the relation between $Q_{1}$ and $\nu_{1}$ in Atoll sources, it can be seen in the right panel of Fig. 4 that, if only the rising part of $Q_{1}-\nu_{1}$ plot is considered, the five Atoll sources form two curve-like tracks, but it is not enough obvious to indicate a clear correlation. We claim that this phenomenon arises from the special physics in the inner disk region for each source, where stellar mass, magnetic field and accretion rate may be the roles of producing them. For the fitting, we fit the three relations to each rising curve. This tentative fitting is not good enough to show a clear correlation although the $\chi^{2} /$ d.o.f. of linear fitting is better than the other two for the curve with relatively low $Q_{1}$ values (formed by 4U 1636-53, 4U 1728-34 and 4U 1735-44), and the $\chi^{2} /$ d.o.f. of the power-law fitting is better to the curve with high $Q_{1}$ values (formed by $4 \mathrm{U}$ 1608-52 and 4U 182030).

Acknowledgements. We acknowledge M. Méndez and D. Barret for providing the data. This work is supported by the National Natural Science Foundation of China (NSFC 10773017, NSFC 10773034, NSFC 10778716, NSFC 11173024), the National Basic Research Program of China (2009CB824800, 2012CB821800), NSC 99-2112-M-007-017-MY3, and the Fundamental Research Funds for the Central Universities. We are very grateful for the anonymous referee for critic comments, which has changed the quality of the paper.

\section{References}

Abramowicz, M. A., Karas, V., Kluzniak, W., Lee, W. H., Rebusco, P.: 2003, PASJ 55, 467

Barret, D., Boutelier, M., \& Miller, M. C.: 2008, MNRAS 384, 1519

Barret, D., Kluźniak, W., Olive, J. F., Paltani, S., Skinner, G. K.: 2005a, MNRAS 357, 1288

Barret, D., Olive, J. F., Miller, M. C.: 2005b, MNRAS 361, 855

Barret, D., Olive, J. F., Miller, M. C.: 2005c, Astron. Nachr. 326, 808

Barret, D., Olive, J. F., Miller, M. C.: 2006, MNRAS 370, 1140

Barret, D., Olive, J. F., Miller, M. C.: 2007, MNRAS 376, 1139

Belloni, T., Mendez, M., Homan, J.: 2005, A\&A 437, 209

Belloni, T., Méndez, M. \& Homan, J.: 2007, MNRAS 376, 1133

Belloni, T., Psaltis, D., van der Klis, M.: 2002, ApJ 572, 392
Boutelier, M., Barret, D., \& Miller, M. C.: 2009, MNRAS 399, 1901

Boutelier, M., Barret, D., Lin, Y., Török, G.: 2010, MNRAS 401, 1290

Hasinger, G., \& van der Klis, M.: 1989, A\&A 225, 79

Hasinger, G.: 1990, RvMA 3, 60

Kluzniak, W., Abramowicz, M. A.: 2001, Acta Physica Polonica B 32, 3605

Méndez, M.: 2006, MNRAS 371, 1925

Méndez, M., van der Klis, M.: 1999, ApJ 517, L51

Méndez, M., \& van der Klis, M. 2000, MNRAS 318, 938

Méndez, M., van der Klis, M., \& Ford, E.C. 2001, ApJ 561, 1016

Miller, M. C., Lamb ,F. K., Psaltis, D.: 1998, ApJ 508, 791

Psaltis, D. et al. 1998, ApJ 501, L95

Psaltis, D. et al. 1999a, ApJ 520, 763

Psaltis, D., Belloni, T., van der Klis, M.: 1999b, ApJ 520, 262

Stella, L. \& Vietri, M.: 1998, ApJ 492, L59

Stella, L. \& Vietri, M.: 1999, PRL 82, 17

Stella, L., Vietri, M., Morsink, S. M.: 1999, ApJ 524, L63

Titarchuk, L. \& Wood, K.: 2002, APJ 577, L23

Török, G.: 2009, A\&A 497, 661

van der Klis, M.: 2000, ARA\&A 38, 717

van der Klis, M.: 2006, A review of rapid X-ray variability in Xray binaries in Compact stellar X-ray sources, W.H.G. Lewin \& M. van der Klis (eds. ), Cambridge University Press, p. 39; (astro-ph/0410551).

van der Klis, M.: 2008, AIPC. 1068, 163

Wang, J., Zhang C. M., Zhao Y. H., Lin Y. F., Yin H. X., Song L. M.: 2011, A\&A 528, 126

Yu W. F., van der Klis M. 2002, ApJ 567, 67

Yu W. F., van der Klis M., Jonker P. G. 2001, ApJ 559, 29

Zhang, C. M.: 2004, A\&A 423, 401

Zhang, C. M., Yin, H. X., Zhao, Y. H., Zhang, F. \& Song, L. M.: 2006, MNRAS 366, 1373 